\title{
Quelques observations sur des fissures radiales dans le bois de douglas
}

\author{
H. POLGE \\ I.N.R.A., Station de Recherches sur la Qualité des Bois \\ Centre de Recherches forestières de Nancy, \\ Chrmpenoux, F 54280 Seichamps
}

\begin{abstract}
Résumé
Un inventaire des fissures radiales à l'état frais a été fait sur radiographies de carottes de sondage de $5 \mathrm{~mm}$ prélevées dans 334 douglas de 13 ans de 11 provenances différentes. Ces fissures se produisent toujours dans le bois initial, et jamais dans les cernes de l'aubier à humidité normale. Les cavités sont vides tant que les cernes à fissures ne sont pas atteints par la duraminisation. La liaison avec la présence de zones sèches à l'intérieur des cernes de l'aubier est peu marquée au niveau des provenances, mais semble plus nette au niveau des années.

L'explication par les facteurs du climat est difficile. La structure du bois initial joue un rôle dans l'expression du phénomène : la corrélation de la fréquence annuelle des fissures est positive et non significative avec la largeur du bois initial, mais négative et très significative avec la densité minimale.
\end{abstract}

\section{Revue sommaire de littérature}

Les fissures dont il va être question correspondent à des fentes radiales de très petites dimensions qui se produisent en grand nombre certaines années, mais sans jamais s'étendre au-delà des limites d'un cerne; il s'agit donc d'un phénomène tout à fait différent des gélivures qui sont rarement très nombreuses sur un même arbre, mais y pénètrent profondément, et intéressent donc toute une série d'accroissements.

C'est, semble-t-il, Pryor (1947) qui les a étudiées le premier sur le pin radiata : il a montré qu'elles s'étendaient de la fin du cerne d'une année au début du bois final de l'année suivante, qu'elles avaient une forme elliptique ou lenticulaire, que leur longueur radiale pouvait varier de 0,8 à $5 \mathrm{~mm}$, qu'elles affectaient surtout les jeunes tiges de moins de $15 \mathrm{~cm}$ de diamètre et étaient plus fréquentes à la base de l'arbre qu'à la partie supérieure; il attribuait leur formation au gel qui pourrait occasionner, pendant la période de dormance, une rupture de l'assise génératrice qui ne retrouverait sa continuité que lors de la formation du bois final.

Un phénomène analogue a été décrit sur le douglas en Nouvelle-Zélande par Reid \& Mrtchell (1951); ils ont observé que, dans cette essence, les fissures sont le plus souvent remplies de gomme, que leur longueur dans le sens du fil du bois 
peut dépasser $15 \mathrm{~cm}$ et qu'elles se trouvent confinées au bois initial des cernes à faible texture; bien quattribuant ces fissures à la sécheresse, ils n’ont pas réussi à établir de liaisons entre leur présence et les conditions climatiques pendant la période de formation du bois initial correspondante.

LuTz (1952) a étudié les fissures radiales de Picea glauca et les a attribuées au collapse des cellules à parois minces du bois initial sous l'effet du déséquilibre entre alimentation en eau et transpiration qui se produit lorsque le sol est gelé.

Amos (1964), travaillant sur le pin radiata, a montré que les fissures se produisaient toujours en limite d'un rayon ligneux, et a pu observer un cas où un morceau de rayon qui manquait d'un côté de la fissure se retrouvait de l'autre; il en déduisait que la fissuration du bois initial n'avait pu se produire que postérieurement à sa formation, donc au plus tôt pendant l'élaboration du bois final de l'année, ce qui expliquerait labsence de corrélation trouvée par les chercheurs précédents quand ils prenaient en considération le climat prévalant en début de saison de végétation. Retenant l'idée d'une influence du froid, il montrait que le coefficient d'expansion du bois frais de pin radiata en fonction de la température étant négatif dans le sens tangentiel, un refroidissement pouvait entraîner une dilatation du bois dans cette direction qui, combinée avec une contraction de la sève, favoriserait le collapse des trachéïdes ; celui-ci se produirait dans les fibres différenciées et à membrane secondaire pleinement formée dont la rigidité s'oppose à ce qu'elles se déforment progressivement sous l'effet de ces changements de dimension, comme c'est le cas pour les cellules très plastiques de l'assise cambiale et de la zone en cours de différenciation.

\section{Observations faites dans l'expérience de douglas d'Amance}

Le dispositif expérimental concerné est décrit par ailleurs dans le présent numéro (pp. 379 à 398). L'examen de 334 carottes de sondage prélevées pour les études sur l'aubier a fait apparaître incidemment une grande abondance de fissures radiales, présentes à la fois sur les radiographies à l'état frais et à l'état sec, et ne pouvant donc pas être attribuées à un phénomène de séchage, et il a paru intéressant d'essayer de profiter de cette occasion pour procéder à quelques observations à leur sujet.

Des prélèvements ne mesurant que $5 \mathrm{~mm}$ dans le sens tangentiel ne constituent certes pas le matériel idéal pour estimer la fréquence d’anomalies anatomiques qui, malgré leur grand nombre, sont souvent distantes les unes des autres, dans cette direction, d'environ $10 \mathrm{~mm}$; mais, cétait la première fois que l'on disposait d'un échantillonnage aussi étendu permettant d'avoir une bonne idée de la variabilité entre arbres, et, d'autre part, s'agissant d'apprécier à l'aide de sondages de direction fixe une particularité de structure se reproduisant avec la même intensité sur toute la circonférence des arbres, il paraît exclu que l'analyse soit biaisée au profit d'une année ou d'un groupe d'arbres particulier.

Une première remarque d'un grand intérêt, qui ne peut être faite que lorsqu'on dispose de radiographies à l'état frais donnant de façon précise la localisation de l'eau dans le bois, s'impose : on n'observe jamais de fissures dans les zones normalement humides de l'aubier, mais seulement dans les parties sèches de celui-ci; ceci est bien illustré par larbre de la figure 1, où l'on trouve des fissures en 1972, 1973, 1975 et 1976, années à zones sèches, mais pas en 1977 et 1978, années à aubier 
normalement riche en eat; qui plus est, le hasard a fait quen 1976 la limite de la partie humide soit en oblique par rapport à celle du bois initial, et la fissure se trouve bien dans la partic de laccroissement où existe une zone sèche! En fait, sur les 54 fissures observées, 53 ont été recensées dans les zones à faible teneur en eau de laubier, et une dans le bois de caxur, car il est bien évident quelles ne disparaissent pas lorsque la duraminisation atteint les cernes où elles se trouvent.

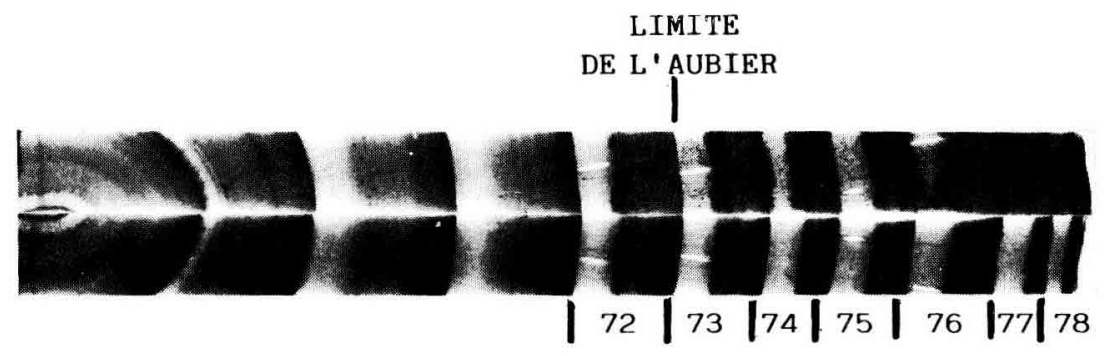

FIG. 1

Positif de radingraphies au grossissement 2 de carottes de sondage à l'état frais (partie supérieure) et à l'état sec (partie inférieure) avec des fissures dans 4 cernes.

Positive print at the magnification 2 of $X$-ray images of $5 \mathrm{~mm}$ cores in wet conditions (upper part) and in dry condition (lower part) showing radial fissures within 4 rings.

Limite de l'aubier : limit of sapwood.

Pour le reste, ce premier examen a confirmé un résultat donné par la littérature. mais en a infirmé un autre :

- les fissures somt bien limitées an seul bois initial, ainsi que le montre netlencent la comparaison des radiographies à l'état frais et à l'état sec.

- mais les cavités, de forme lenticulaire très aplatie, sont vides, et non remplies de gommes ou de résine, ce qui résulte également très clairement du noircissement de leur image radiographique (positif correspondant parfaitement blanc), et est confirmé par l'examen microscopique, la seule exception étant fournie par la fissure se trouvant déjà en zone duraminisée.

Linventaire chiffré des fissures est donné ci-dessous à titre indicatif :

\begin{tabular}{|c|c|c|c|c|c|c|c|c|c|c|c|}
\hline Provenances & 1 & 2 & 3 & 4 & 5 & 6 & 7 & 8 & 9 & 10 & 11 \\
\hline $\begin{array}{c}\text { Nombre d'arbres à tissu- } \\
\text { res } \ldots \ldots \ldots \ldots \ldots \ldots \ldots\end{array}$ & 1 & 3 & 8 & 6 & 4 & 3 & 3 & 6 & 4 & 2 & 3 \\
\hline $\begin{array}{l}\text { Nombre d'années à fissu- } \\
\text { res } \ldots \ldots \ldots \ldots \ldots \ldots . .\end{array}$ & 1 & 4 & 4 & 5 & 4 & 4 & 1 & 2 & 2 & 2 & 3 \\
\hline $\begin{array}{c}\text { Nombre de cernes à fis- } \\
\text { sures } \ldots \ldots \ldots \ldots \ldots \ldots \\
\end{array}$ & 1 & 5 & 9 & 11 & 4 & 5 & 2 & 7 & 4 & 2 & 4 \\
\hline
\end{tabular}


En outre, les 43 arbres à fissures se répartissent à raison de 24 dans la partie du dispositif irriguée en 1976 et 1977 et 19 dans la zone témoin soumise de plein fouet aux effets de la sécheresse de 1976. Celle-ci n’a donc manifestement pas eu un effet positif sur lapparition des fentes.

La liaison avec la fréquence des zones sèches n'est, au niveau infraspécifique, pas très marquée : ainsi les provenances 5 et 7 , qui avaient le plus grand nombre d'arbres à zones sèches sont ici assez mal classées; il convient cependant de noter que la provenance 4 , qui présente le plus d'années et le plus de cernes à fissures, arrivait également en tête pour ces deux mêmes caractéristiques quant à la fréquence des zones sèches. Au total, bien quaucune fente ne se produise en labsence de ces zones à faible humidité, on peut donc penser qu'inversement leur abondance dans une provenance ne signifie pas automatiquement qu'elle soit particulièrement sensible al phénomène de fissuration.

La répartition par années des fissures est la suivante :

\begin{tabular}{c|c|c|c|c|c|c|c}
\hline Années & 1972 & 1973 & 1974 & 1975 & 1976 & 1977 & 1978 \\
\hline $\begin{array}{c}\text { Nombre de fissu- } \\
\text { res ........ }\end{array}$ & 9 & 18 & 5 & 14 & 4 & 2 & 2 \\
\hline
\end{tabular}

Ici, la liaison avec la présence d’un bois initial sec dans l'aubier paraît meilleure, et, en particulier, les deux années à plus fort taux de fissuration, 1973 et 1975, sont également, mais dans loordre contraire, celles où less zones sèches sont les plus fréquentes.

Cependant, comme pour ces dernières, et en conformité avec la littérature, l'étude des relations avec les facteurs du climat savère décevante; lexplication par le froid est contredite par labondance des fissures en 1975 : le temps a été exceptionnellement clément durant toute la période de dormance 1974-1975, avec une température moyenne de novembre 1974 à avril 1975 inclus supérieure de 1,8 degré à la normale, et un nombre de jours de gelée durant les mêmes mois de 60 contre une moyenne sur 15 ans de 83,7. La prise en considération de la période de croissance ne conduit pas à de meilleurs résultats, puisque, après les mois de mai et juin à températures normales, ceux de juillet à septembre, qui correspondent en gros à la période de formation $\mathrm{du}$ bois final, ont à nouveau été relativement chaud $(+1,3$ degré par rapport à la moyenne). L’année 1973, qui détient le record des fissurations, se rapproche beaucoup plus de la moyenne, mais en étant, au contraire, un peu plus froide.

On pourrait également penser à une influence possible de la sécheresse, en observant que celle de 1976 a suivi immédiatement la production du cerne 1975 à forte fissuration, et que le déficit en eau a commencé à se produire au dernier trimestre 1975 (-38,7 mm) et au premier trimestre 1976 (-62 mm), mais, là encore, l'explication ne s’appliquerait pas à 1973 qui, au contraire, a été suivie d'une année à pluviosité nettement supérieure à la normale.

Ces tentatives pour trouver l'origine première des fissures radiales s'avérant vaines, on est tenté de rechercher des facteurs de structure du bois qui permettraient 
de relier la cause à l'effet, c’est-à-dire qui faciliteraient l’expression du phénomène. Il est en effet logique de penser que, quels que soient les facteurs de lenvironnement qui sont responsables de ces fissurations, elles ne peuvent au niveau final résulter que de contraintes tangentielles dont les effets peuvent varier en fonction des caractéristiques du bois sur lequel elles s'exercent, ćest-à-dire, au cas particulier, du bois initial.

La littérature ayant mentionné à plusieurs reprises une influence possible de la largeur de cette zone initiale, un premier calcul de corrélation a été fait entre celle-ci et le pourcentage d'arbres ayant des fissures (après transformation en arc sinus racine) pour les 7 années disponibles; il a permis de confirmer les observations non chiffrées de la bibliographie, puisque, s'il n'est pas significatif, le coefficient est cependant positif $(r=+0,45)$, et l'on a donc bien une tendance à avoir plus de fissures les années où le bois initial est plus large.

Cependant, en mécanique, la résistance d'un ensemble hétérogène est conditionnée par celle du point où elle est la plus faible; celui-ci coïncidant, sclon toute vraisemblance, avec le point de densité minimale à l'intérieur du cerne annuel. il était logique de calculer la corrélation entre elle et le pourcentage d'arbres à fissures (toujours après transformation); on trouve (voir figure 2) :

$$
r=-0,88: *
$$

La liaison, très significative pour 5 degrés de liberté, est, comme on pouvait le prévoir, négative, et, par conséquent, les annćes à faible densité minimale annuelle présentent un risque accru de fissuration.

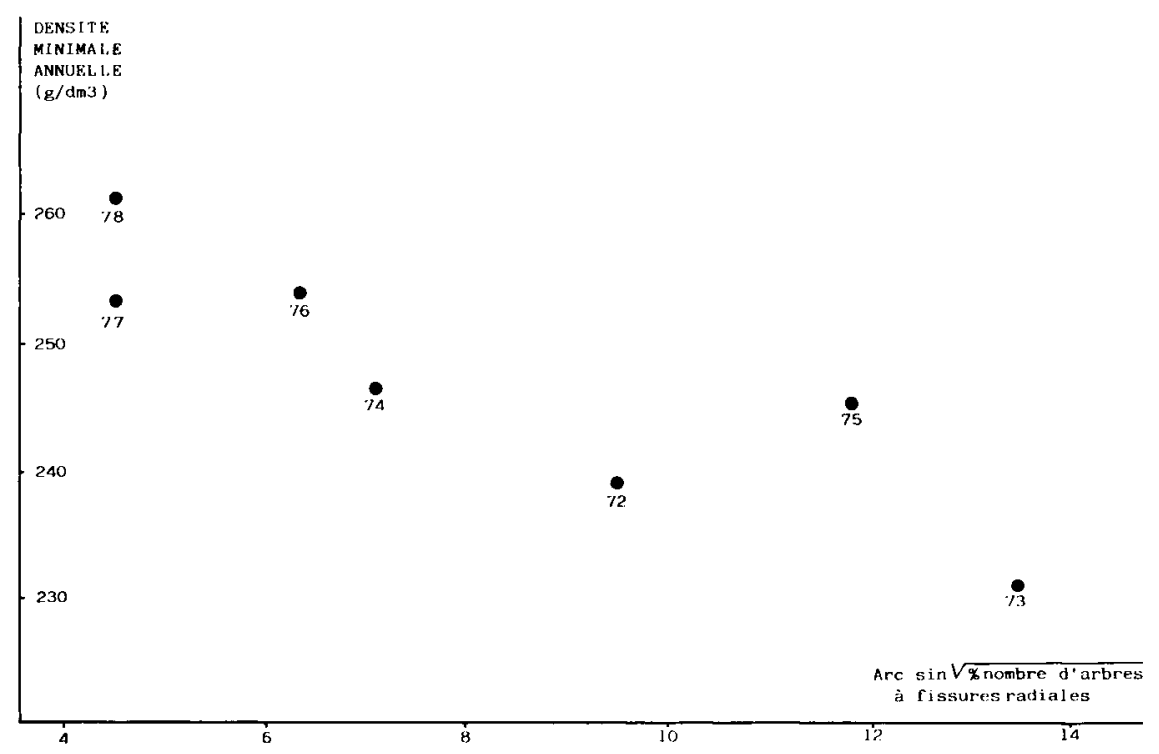

FIG. 2

Corrélation entre la densité minimale annuelle et la fréquence des fissures radiales. Correlation between minimum annual density and the frequency of radial fissures. Nombre d'arbres à fissures radiales : number of trees with radial fissures. 


\section{Conclusion}

Malgré une certaine inadéquation du dispositif expérimental dune part, et de l'échantillonnage d'autre part, les observations dont il est rendu compte ci-dessus ont permis dapporter quelques indications nouvelles sur les fissures radiales des arbres sur pied, en montrant que, si elles sont limitées au bois initial, elles ne se produisent que lorsque celui-ci est anormalement sec, que les liaisons avec les facteurs du climat précédemment mis en cause ne sont pas certaines, et surtout en mettant en évidence une influence de la densité minimale annuelle beaucoup plus nette que celle de la largeur du bois initial. Mais l'essentiel du problème reste à résoudre.

En dehors de l'intérêt qu il présente pour la biologie de laarbre, on peut šinterroger sur son importance économique : il ne faut, semble-t-il, ni la sous-estimer, ni la surestimer :

Amos (1954, op. cit) a justifié son étude par les inconvénients graves que ce défaut entraîne pour le pin radiata employé en placages; or, il est logique de penser que l'on cherchera de plus en plus en France à dérouler pareillement des résineux, et, en particulier des douglas; par ailleurs, lindustrie s'efforce, pour des raisons de rentabilité, d'utiliser des arbres toujours plus petits, et aussi, de réduire le plus possible le diamètre des noyaux de déroulage. Pour toutes ces raisons, les conséquences technologiques défavorables d'une anomalie de structure présente seulement dans les jeunes arbres ne peuvent que prendre de l'importance à l'avenir.

C'est cependant la première fois que ce défaut est observé sur des bois français, et notamment sur le douglas; cela ne veut pas dire qüil n’existe pas ailleurs qu’à l'Arboretum d'Amance, car ni le sol, ni le climat de celui-ci ne s'écartent beaucoup de ceux de nombreuses zones de reboisement; en fait, il est probable que la radiographie a permis de voir ce qu'un examen superficiel non attentif du bois, surtout non raboté, naaurait pas révélé.

Il convient done de rester vigilant, et de profiter des expériences futures pour essayer de connaître l'étendue du phénomène des fissures radiales sur les résineux français, de préciser la hauteur et le diamètre de la partie de grume concerné et, à partir du moment où l'on disposera de résultats pour un nombre suffisant d'années et de situations écologiques, d'en analyser le déterminisme.

Reşu pour publication en octobre 1981.

\section{Summary}

\section{Some observations about radial fissures in douglas fir}

Radial fissures in wet conditions have been numbered on X-rays images of $5 \mathrm{~mm}$ cores taken from 334 douglas firs of 11 provenances at the age 13. Fissures always occur in the early wood, but never in the normally wet sapwood rings. They are empty before heartwood formation. The relationship with dry areas within these rings is poor at the provenance level, but seems better at the year level. 
It is difficult to explain the phenomenon by the climatic factors, but the wood structure of the carly wood plays a role : the correlation with its width is positive and non significant, that with the minimum annual density is negative and very significant.

\section{Références bibliographiques}

Amos G.L., 1954. Radial fissures in the early wood of conifers. Aust. J. Bot., 2, 22-34. Lurz H.J., 1952. Occurrence of clefts in the wood of living white spruce in Alaska. J. For., $50(2), 99$.

Pryor L.D., 1947. Damage to Pinus radiata by climatic agents. Austr. For., 11. 57.

Reid J.S. et Mischel. L.T., 1951. Internal checks occurring in Douglas fir from two sites. For. Prod. Res. Notes (N.Z.), 1 (2), 2. 